\title{
Ecologias híbridas: interações entre organismos artificiais e naturais em ambientes telemáticos
}

GUTO NÓBREGA

\section{Resumo}

A presente comunicação trata do registro e análise de dois projetos em arte telemática realizados em 2011 que tiveram a participação do NANO - Núcleo de Arte e Novos Organismos - Escola de Belas Artes - UFRJ, laboratório de pesquisa coordenado pelos professores Dr. Carlos (Guto) Nóbrega e Dra. Maria Luisa Fragoso, vinculado ao Programa de Pós Graduação em Artes Visuais. Ambos projetos envolveram a criação de sistemas artificiais para interatividade em ambientes telemáticos. $\mathrm{O}$ texto apresenta pontos relevantes nos dois projetos, suas relações, ressonâncias e desdobramentos. $\mathrm{O}$ foco de nossa análise se encontra no processo de invenção de interfaces artificiais, suas hibridações, complexidade e modos de interação e presença no contexto de trabalhos de arte telemática.

Palavras-chave: Interação, presença, telemediação 


\title{
Hybrid ecologies: interactions between artificial and natural organisms in telematic environments
}

GUTO NÓBREGA

\begin{abstract}
This paper reports and analyses two projects in telematic art realized in 2011 that had the participation of NANO - Nucleus of Art and New Organisms - School of Fine Arts - UFRJ, research laboratory coordinated by Dr. Carlos (Guto) Nobrega and Dr. Maria Luisa Fragoso, as part of the Post Graduate Program in Visual Arts. Both projects involved the creation of artificial systems for interactivity in telematic environments. The text will present relevant points of the two projects, their relations, resonances and unfoldings. The focus of our analysis is the process of invention of artificial interfaces, their hybridizations, complexity and modes of interaction and presence in the context of works of telematic art.
\end{abstract}

Keywords: Interaction, presence, telemediation 


\section{Introdução}

No ano de 2011, o núcelo laboratorial NANO foi contemplado em dois projetos de pesquisa e desenvolvimento de interface realizados em colaboração com universidades no Brasil e no exterior. Os projetos "Laboratorium Mapa D2", idealizado e coordenado pela Profa. Dra. Ivani Santana, da Universidade Federal da Bahia e o Projeto "Ecotelemedia", idealizado pelo Dr. Kjell Yngve Petersen da IT University of Copenhagen, Dinamarca, tiveram em comum o foco na articulação de processos colaborativos com base em redes telemáticas para pesquisa de performances assistidas pelas tecnologias da informação e comunicação. Nos próximos parágrafos trataremos de cada um desses projetos individualmente para em seguida apresentarmos suas ressonâncias e análise.

\section{Laboratorium Mapa D2}

O projeto Laboratorium Mapa $\mathrm{D}^{1}$ envolveu diversos grupos de pesquisa de instituições acadêmicas nacionais, entre os quais figuram o: NANO/UFRJ, Telemidia/PUC RJ, GP Poética/UFBA, Computação/ UFBA, LPCA e Grupo de Pesquisa Computacional/UFC, e LAVID/UFPB. Cada núcleo artístico (grupos da Universidade Federal da Bahia, Fortaleza e Rio de Janeiro) trabalhou em diálogo com grupos de tecnologia, responsáveis pela articulação das redes de interconectividade para transmissão de áudio e vídeo em tempo real com base na ferramenta Arthron ${ }^{2}$ desenvolvida pelo LAVID/UFPB. O propósito maior desse consórcio entre laboratórios de pesquisa foi a investigação sobre o potencial das tecnologias da informação e comunicação para a criação de produtos artísticos. Para tanto foram realizados com os demais participantes, ao 
longo de 2011, cerca de 20 encontros virtuais; 4 Open Labs abertos a interação com os usuários-web e público em geral; 3 workshops presenciais sobre o processo criativo e estrutura telemática, 1 mostra parcial, 1 apropriação criativa e 1 mostra final sob o titulo "Frágil" realizada no MAM-RJ em 1o de dezembro de 2011 durante o evento "Desafios da Arte em Rede". "Frágil" precedeu o Festival Internacional de Cultura Digital no Rio de Janeiro.

\subsection{HA}

O papel do NANO nesse processo foi a criação e implementação de uma interface robótica com capacidade para interação local e remota com os performers e o público. O robô batizado de HA, acrônimo para "Hiperorganismo ${ }^{3}$ Antropofágico", foi proposto ao grupo como potencial interface para o projeto. O HA resultou da adaptação e implementação de uma estrutura previamente imaginada como híbrido entre planta e sistema artificial, cuja função maior seria possibilitar uma visualização, em seu próprio corpo, das atividades eletrofisiológicas da planta. Essas visualizações (data visualization) seriam projetadas na estrutura do híbrido através do uso de um micro projetor e uma membrana pulsante como parte de sua estrutura. Esse esquema inicial derivou da pesquisa de doutoramento desse autor sobre hibridação entre plantas e sistemas artificiais, cujo processo resultou na obra artística "Breathing"4. Para o projeto Laboratorium Mapa D2 o HA foi concebido na forma de um robô cujas funções implicam:

- Cabeça com capacidade de visão artificial monocular;

- Pescoço com capacidade de locomoção em quatro sentidos: para os lados (direita e esquerda), para cima e para baixo;

- Estrutura para projeção de imagens na forma de bolsa de ar automatizada para contrair e expandir segundo parâmetros externos e/ou internos ao hiperorganismo;

- Sensores de distância para mapeamento do ambiente e interações;

- Capacidade de conexão com internet via protocolos de rede;

- Capacidade para receber e projetar streaming de áudio e vídeo.

Na figura abaixo podem ser visualizados o esquema original do HA e sua forma final apresentada no evento "Frágil". 


\section{hiperorganismo antropofágico}

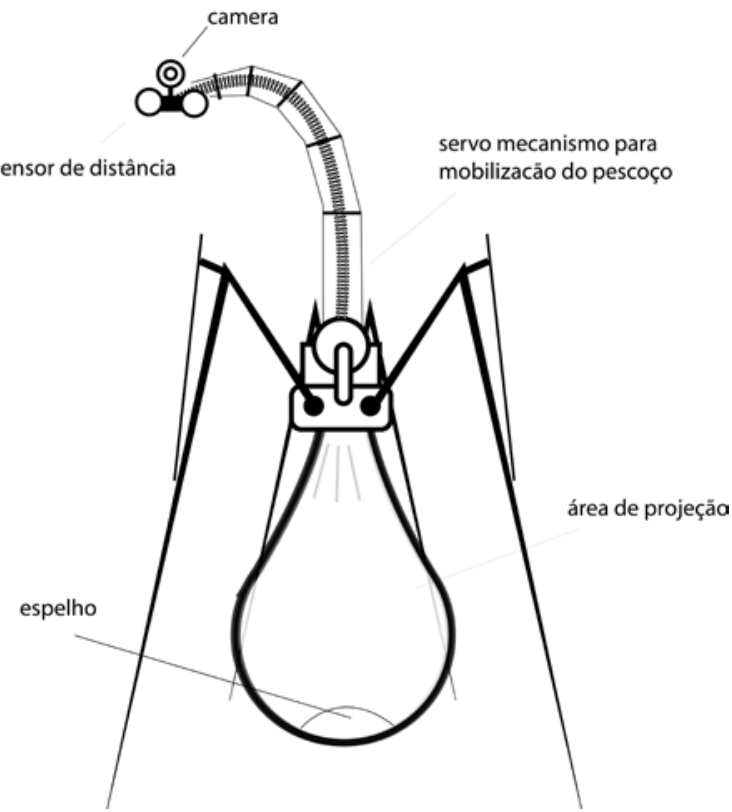

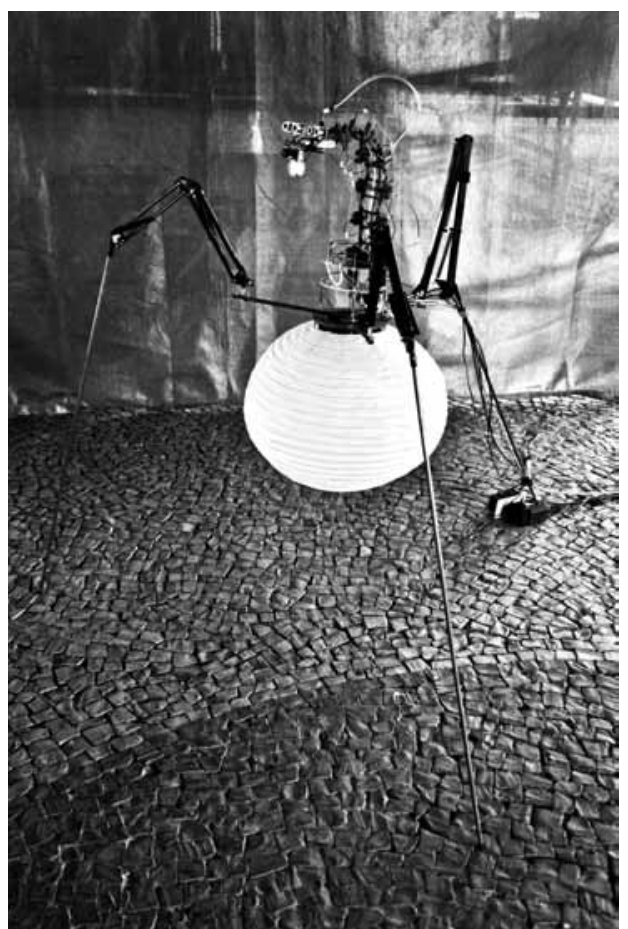

As funções automatizadas desse robô são coordenadas por um microcontrolador do tipo Arduino (2006), associado a um módulo para transmissão de dados sem fio por rádio frequência.

\subsection{Interfaces vestíveis}

Em paralelo ao HA foram desenvolvidas três interfaces vestíveis. $\mathrm{O}$ propósito dessas interfaces foi permitir a interconectividade entre robô e performers. Uma das interfaces serviu para o monitoramento das dilatações do tórax de um dos bailarinos, assim como o envio desses dados para o HA de maneira a controlar as contrações e expansões de sua bolsa de ar. A medida que o bailarino acelerava ou desacelerava sua respiração, movimentos correspondentes podiam ser percebidos no corpo do HA que contraia ou expandia sua bolsa de ar (uma lanterna japonesa) que se asemelhava a um pulmão artificial. Uma segunda interface foi desenvolvida para o monitoramento da intensidade sonora vocal de um dos performances. Por
Figura 1

Esquema original do $H A$ e sua concepção final apresentada no evento "Frágil-RJ. 
meio dessa interface, baseada num microfone eletreto, amplificador operacional, Arduino e transmissor de radio freqüência, as variações de intensidade sonora transformadas em dados numéricos puderam ser enviadas via rede para uma terceira interface, uma veste capaz de receber tais informações e transmutá-las em vibração através de micro-motores. Esses micro-motores (atuadores) espalhados em 4 pontos estratégicos da veste (pescoço, tórax e região abaixo do umbigo) permitiram ao performer sentir em seu próprio corpo a intensidade de voz de um outro performer, criando uma espécie de experiência sinestésica. Uma série de interfaces programadas em Processing permitiram uma mapeamento em tempo real dos fluxos de dados que circulavam entre bailarinos e robô e sistema ${ }^{5}$.

\subsection{Ambiente telemático}

Figura 2 Na figura 2 apresentamos um diagrama aproximado dos fluAmbiente telemático "Frágil-RJ xos de dados, imagem e áudio em transito no evento "Frágil".

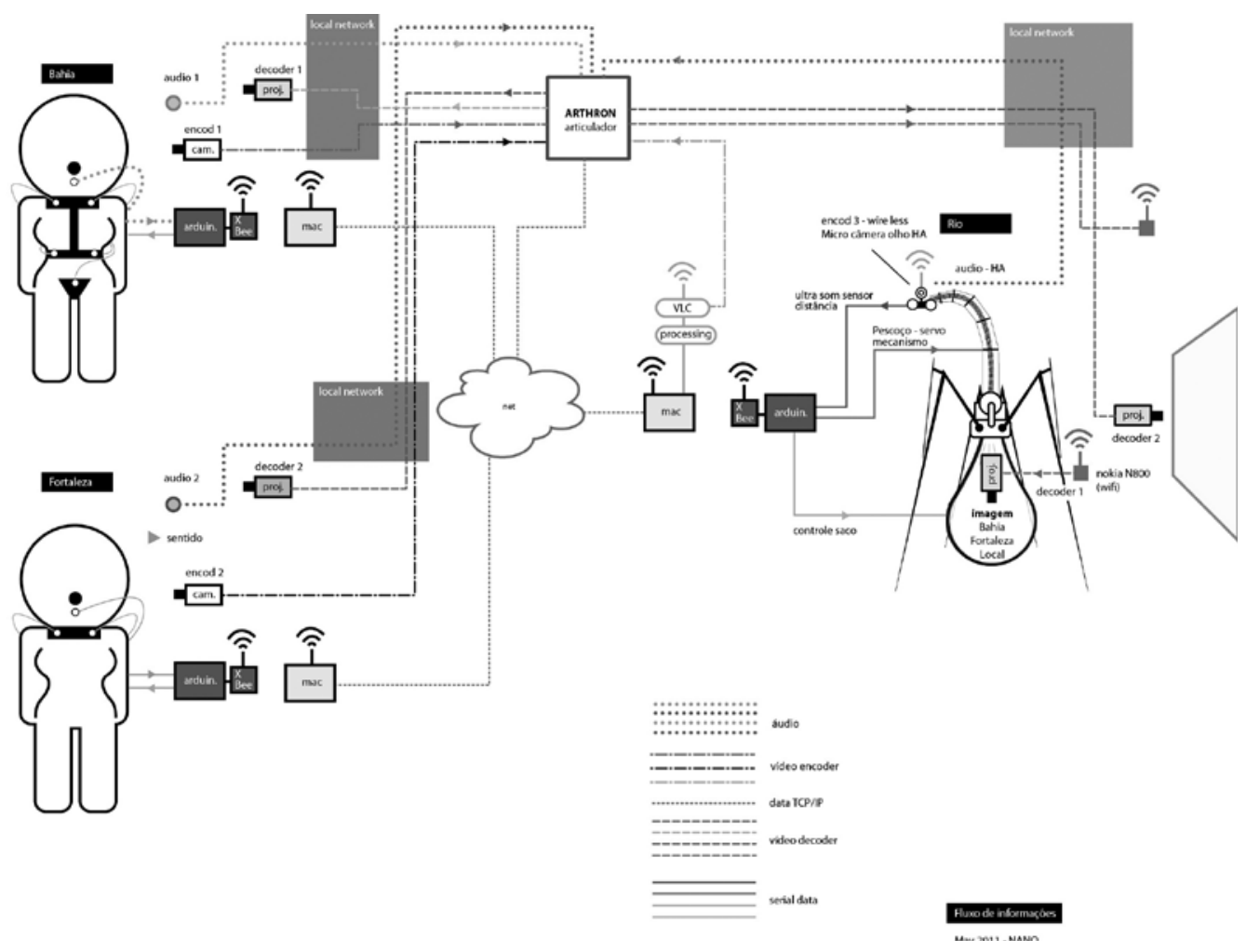


A ideia inicial era que cada grupo artístico se mantivesse em local de origem (Bahia, Rio de Janeiro e Fortaleza) com suas respectivas telas, sistema sonoro e interfaces interativas para construção do evento telemático. Devido a oportunidade da apresentação no MAM-RJ, o grupo da Bahia, coordenado pela Profa. Dra. Ivani Santana, instalou-se no Rio de Janeiro onde atuou localmente com o HA. Apenas o grupo de Fortaleza e sua equipe, sob coordenação do Prof. Hector Briones, Profa. Dra. Walmeri Ribeiro e Profa. Dra. Juliana Rangel permaneceu em seu local de origem, Teatro Municipal de Fortaleza. Remotamente esse grupo se manteve conectado ao Rio de Janeiro via a ferramenta Arthron e protocolo OSC para transmissão/recepção de dados entre as interfaces vestíveis e o robô. A seguir trataremos do projeto "Ecotelemedia", suas interfaces, processos, para então prosseguirmos com uma reflexão sobre os dois projetos, ressaltando suas ressonâncias e tecendo considerações a respeito da interação com interfaces artificiais em ambientes de arte telemática.

\section{Ecotelemedia}

O projeto internacional "Ecotelemedia" resultou do convite feito ao NANO para integrar uma rede de pesquisa colaborativa concebida sob o titulo "The aesthetics of global connectivity: exploring design strategies and networked technologies of distributed sites through artistic processes". Essa pesquisa, que se estendeu ao longo de 2011, reuniu a Universidade Federal do Rio de Janeiro, a IT University of Copenhagen e Central Conservatory of Music, Beijing - China num processo colaborativo coordenado respectivamente pelos pesquisadores Dr. Carlos Nóbrega (Brasil), Dr. Kjell Yngve Petersen (Dinamarca) e Dr. Kenneth Fields (China). Em abril de 2011 os artistas pesquisadores dos três grupos envolvidos no projeto, assim como os demais colaboradores dessa rede, se reuniram no âmbito do laboratório NANO e dependências da Escola de Belas Artes- UFRJ, para discussões metodológicas, oficinas e participação no simpósio "Telemediações. Explorando paradigmas estéticos em ecologias híbridas", organizado para o evento. O objetivo maior desse trabalho exploratório foi estabelecer metodologias de pesquisa e criação artística, substanciadas por trocas locais e telemáticas entre artistas e pesquisadores dos países envolvidos no processo. Para tanto foram realizados encontros, seminários e oficinas. A pesquisa teve como foco os paradigmas estéticos emergentes em ambientes telemati- 
camente mediados. Ênfase foi dada ao experimentalismo com sistemas naturais e artificiais, a experiência participatória e interativa através da performance, imagem e interfaces acústicas, e a investigação do caráter híbrido (natureza e artifício) de uma possível ecologia em curso.

\subsection{Interface híbrida}

A proposta do NANO para o projeto "Ecotelemedia" foi a sonificação de um híbrido composto de planta e sistema artificial programado para transformar variações de condutividade elétrica nas folhas vegetais ${ }^{6}$ em dados numéricos. O coração desse sistema é baseado em um medidor de respostas galvânicas (GSR - Galvanic Skin Response) $)^{7}$ tal qual empregado na obra "Breathing", em que um dos modos de interação com o sistema se dava através do ato de respirar nas proximidades da planta. Para o projeto "Ecotelemedia" foi desenvolvida uma interface na qual três plantas funcionaram como uma espécie de sensor orgânico, gerando três canais de dados variáveis que foram usados no processo de sonificação. Um Led RGB ${ }^{8}$ foi utilizado na monitoração visual das interações com o híbrido. A integração desse sistema ao ambiente telemático e sua sonificação será tratado a seguir.

\subsection{Ambiente telemático}

Essencialmente o ambiente Ecotelemedia foi pensado como um sistema telemático otimizado para performance no qual múltiplos agentes (humanos, maquínicos, vegetais) pudessem interagir de forma relativamente coerente num dado espaço de tempo. Esse ambiente foi constituído de telas LCDs de 40 polegadas, projetores, sistema de áudio, microfones, filmadoras HD, infra-estrutura de rede, computadores Apple, Ipads, Ipods, Cilia ${ }^{9}$ e o sistema híbrido criado pelo NANO. A figura 3 mostra uma esquematização geral dessa estrutura.

Podemos ver nessa imagem o sistema estruturado para operar telematicamente com conexões entre cinco pontos remotos: Brasil (Nano - UFRJ e UFBA), Whashington USA, IT University of Copenhagen e Conservatory of Music, Beijing - China. Podemos afirmar que um dos fatores de coesão centrais ao projeto foi o feedback sonoro gerado no sistema. A ambientação sonora de "Ecotelemedia" se fez pelo 
arranjo digital de múltiplas fontes de dados variáveis para o controle de máquinas sonoras programadas em Pure Data. Os fluxos de dados entre os demais sistemas circularam em rede com base em protocolo $\mathrm{OSC}^{10} \mathrm{e}$ a interconectividade de áudio entre Brasil, Dinamarca e China se deu via Jack Trip ${ }^{11}$. As fontes de dados utilizadas foram: acelerômetros de iPad e iPod manipulados gestualmente pelas duas performances (Ivani e Annika); dois sistemas híbridos (Planta e GSR, cada sistema monitorando três plantas agrupadas), um no Brasil e outro na Dinamarca; um controlador digital Cilia.

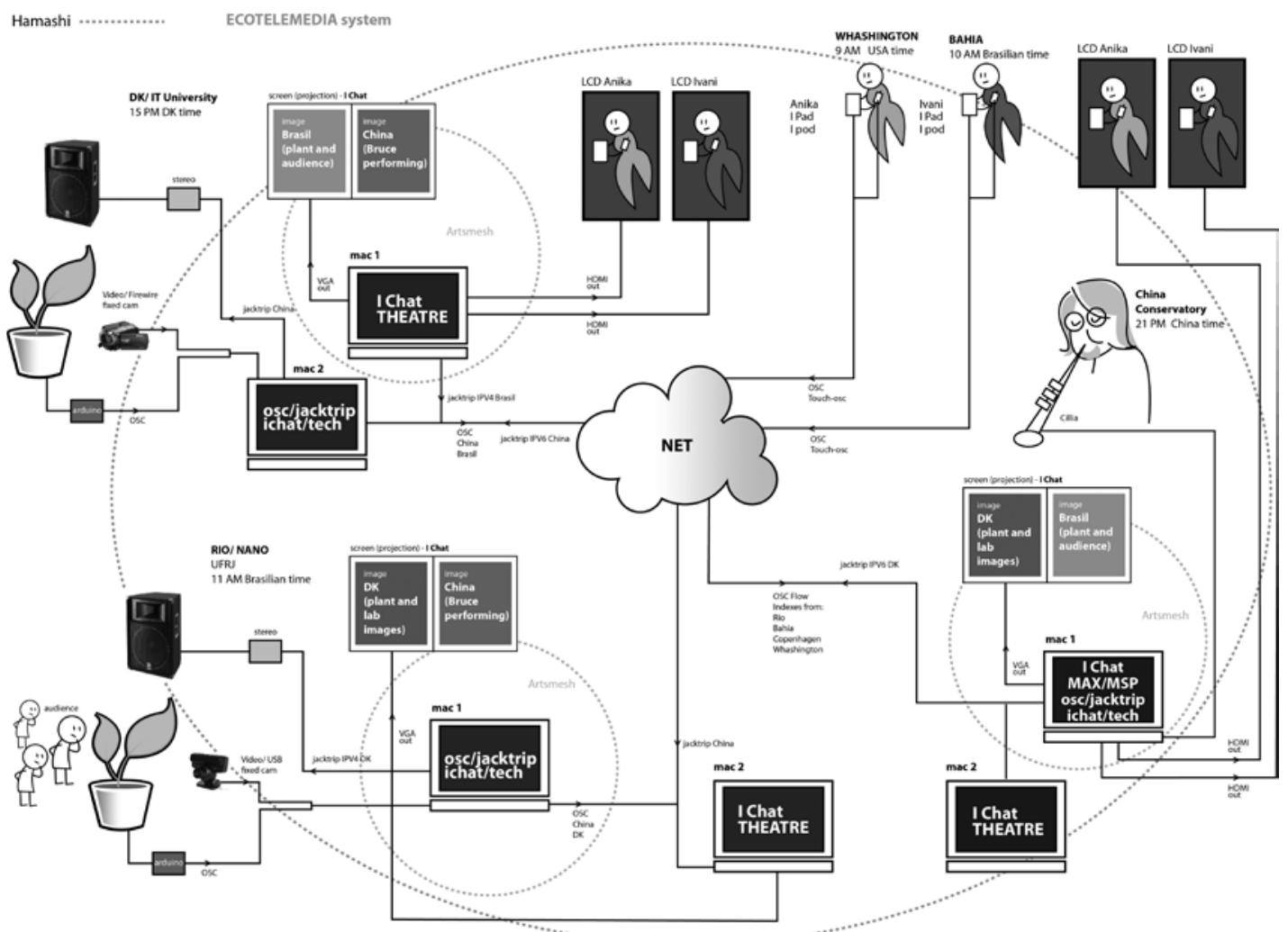

Na semana dos dias 5 a 9 de setembro, num dos laboratórios da IT University of Copenhagen, foi realizada uma oficina intensiva que culminou com a primeira apresentação do Evento Ecotelemedia ao público. Nessa apresentação todos os agentes participantes do evento atuaram no mesmo espaço físico, interconectados por uma rede local. No
Figura 3

Ambiente telemático - Ecotelemedia 
dia 25 de outubro de 2011 a performance ocorreu telematicamente entre países via internet. A seguir faremos uma breve análise dos eventos apresentados.

\section{Ecologia de materiais}

Tem sido um ponto de partida do grupo de pesquisa NANO, no que se refere a sua incursão na invenção de organismos artificiais, a manutenção de uma perspectiva artística e do caráter exploratório, experimental e inovador no processo criativo. Esse contexto artístico no qual o processo de invenção ocorre determina a base para o emprego de certa tecnologia, seu alcance, limites funcionais e conceituais. Partimos do princípio que o curso tomado num dado processo de invenção deve ser vigiado por um olhar atento às variáveis que surgem ao longo do caminho, buscando-se o equilíbrio entre a indeterminação criativa e as certezas e exatidões dos algoritmos e mecanismos empregados nos processos assistidos por tecnologias. Cabe considerar aqui o olhar visionário do filósofo Vilém Flusser (2002) para quem o coeficiente de liberdade criativa nos processos assistidos pela programabilidade tecnológica está diretamente relacionado a capacidade de se manter as vias do experimentalismo abertas. Experimentalismo = liberdade criativa. Nesse sentido, a busca por materiais inventivos que venham cumprir as funções necessárias de um determinado mecanismo, assim como a observação da tecnicidade ${ }^{12}$ (Simondon, 1989) inerente aos materiais empregados, podem e devem orientar tomadas de decisões não antecipadamente determinadas.

Por exemplo, no caso específico do HA o desenvolvimento de sua bolsa de ar, previamente imaginada com base em tecido translúcido inflável ganhou uma outra dimensão ao se empregar uma luminária de papel japonesa como corpo. Em tais processos é comum determinada estrutura conduzir ao acoplamento dessa a uma outra e assim sucessivamente. Isso ocorre, por exemplo, com a forma anelar da lanterna japonesa que levou a um movimento vertical de expansão e contração e consequentemente determinou um certo acoplamento da ventoinha instalada em sua "boca". A ventoinha, adaptação de um cooler de computador, foi responsável por sugar o ar interno e fazer a lanterna se contrair ou expandir (ligar ou desligar a ventoinha) dando a impressão de um organismo pulsante como pulmões de ar. Devido ao posicionamento da ventoinha o mesmo ar sugado 
da lanterna é aplicado para resfriar o transistor de potencia que a controla. É dessa organização coerente entre partes e funções que deriva a forma desse hiperorganismo. Sua base estrutural advém do reaproveitamento de objetos técnicos inicialmente pensados para outras funções mas que ganharam uma ressignificação num novo corpo inventado. A poética em torno do HA passa pela fruição desses acoplamentos inusitados e inventivos que lhe dão forma. Similarmente, no caso do híbrido criado para o projeto Ecotelemedia, são os acoplamentos, ora impensados, que acabaram por tornar visível o invisível. A planta conectada ao sistema transdutor de sinais elétricos em dados numéricos e luz engendram um só corpo, estranho em sua natureza híbrida.

\section{Coerência e interação}

Uma das questões que motivaram a criação do HA para o projeto "Laboratorium Mapa D2", tanto quanto a criação do híbrido para o projeto "Ecotelemedia", foi a indagação sobre a presença e participação de um agente artificial robótico no contexto de um ambiente performático telemediado. As questões mais comuns inerentes ao projeto foram articuladas ao redor do modo de existência de tais objetos técnicos, suas relações com os demais agentes da performance e evidentemente o público. O que nos faz presente num determinado ambiente? Quais formas de presença podem ser pensadas no contexto de agentes artificiais telemáticos? De quais maneiras podemos considerar a idéia de presença no contexto de uma possível ecologia de estruturas artificiais em evolução? Um sistema robótico certamente aponta para questões relacionadas a sua própria existência como criatura num dado ambiente, especialmente tratando-se de um espaço voltado para uma experiência artística. O evento Frágil foi muito positivo em contextualizar tais questões.

Na apresentação do evento Frágil no MAM-RJ reunimos bailarinos, músicos e demais estruturas de dados e redes para criar um ambiente no qual, por alguns minutos, se estabelecesse o espaço ideal no qual o público pudesse participar da experiência de um evento telemático. Esse ambiente foi construído por imagens, sons e ações de diversas ordens e modalidades. Abaixo da camada de eventos performados pelos bailarinos sob a direção segura da idealizadora do projeto, a artista pesquisadora Ivani Santana, uma subliminar camada de protocolos de rede e fluxos de dados deram cor- 
po a dimensão imaterial e tecnológica do evento. Foi nesse contexto que se deu a participação do HA no "Frágil".

Com movimentos aleatórios de seu pescoço, e análise de profundidade de campo através de sensores de distância ultrasônicos, o robô foi capaz de identificar quando algo se aproximava de seu campo visual e fixar seu "olhar" neste objeto ou corpo. Uma grande tela próxima ao HA apresentava o que chamamos de sua "visão subjetiva". Fibras óticas respondendo luminosamente aos sensores de distância permitiram uma outra camada de visualização das interações do HA com o seu redor. Através de comunicação via rede, o robô foi capaz de contrair e expandir sua bolsa de ar conforme os movimentos de tórax de um dos bailarinos do evento. Essa relação foi apresentada de maneira clara ao público presente através de vídeo projeções. No entanto, apesar das correlações funcionais do HA, sua inserção física no ambiente local e telemático (imagens enviadas em tempo real para o teatro em Fortaleza), ficou notável uma sensação de incompatibilidade. As funcionalidades do robô, sua autonomia, seu modo de expressão não foram suficientes para "integrá-lo" por completo naquele ambiente. Apesar das interações com o público, ainda que tímidas por parte daqueles que se aproximavam, ou mesmo com os bailarinos, as mesmas não nos pareceram suficientemente consistentes para torná-lo "presente". Acreditamos que tenha faltado tempo de convívio, ensaio (assumindo essa falta como parte da complexidade do processo de dar corpo a uma pesquisa e evento dessa ordem), para que as idiossincrasias do HA, assim como sua sensibilidade artificial perante o público pudessem ganhar contornos mais visíveis. Nos parece que a questão de presença se passa pela noção de coerência, relevante a organização dos seres vivos (Ho, 1993). Presença nos parece implicar, de certa maneira, algum tipo de integração orgânica ao sistema do qual se faz parte. Estamos presentes muitas vezes sem sermos notados. Nos parece então que a capacidade de oscilar entre presença e ausência, capacidade essa intrínseca aos vivos, é essencial para nos colocar em certo lugar de evidência como agente num dado sistema. Vem daí a importância da noção de presença para se pensar um ecologia híbrida de agentes maquínicos e naturais em contextos telemáticos.

No evento "Ecotelemedia" o som foi um elemento importante para a noção de integração e coerência do híbrido e demais agentes no ambiente telemediado. Ao respirar próximo as plantas que se localizavam, um grupo, na Dinamarca e 
outro no Brasil, os dados gerados pelo sistema alteravam a cor do Led RGB localmente ao mesmo tempo que variáveis eram enviadas via rede para a China. Os dados recebidos na China foram mixados aos demais fluxos numéricos e sonificados através dos controles da Cilia e Pure Data. Dessa maneira, ao interagir com um híbrido no Brasil, recebia-se em tempo real um feedback sonoro, que também se fazia presente nos demais países envolvidos na performance. A noção de feedback, o ato de respirar junto ao híbrido, a visualização dos dados através de projeções e a troca constante entre os demais integrantes do evento através de $i$-Chat, foram elementos importantes para emergência de coerência no sistema e a construção de interconectividade entre seus agentes. Sempre que por questões técnicas um desses parâmetros deixou de funcionar o sistema entrou em estado de desordem e caos e a interação entre as demais partes se apresentou comprometida ${ }^{13}$.

\section{Conclusão}

Performances telemáticas, cujos integrantes não encontramse situados numa mesma localização física, parecem esbarrar num problema comum. Como tornar claro para a audiência o caráter telemediado da obra? Como fazer entender, sem uma prévia descrição do que está por acontecer, que os atores se encontram remotamente localizados, que as imagens, fluxos de dados e áudio são em tempo real e que a obra emerge dessa topologia para além da fisicalidade dos espaços interconectados? Quanto aos agentes envolvidos, qual o papel dos sistemas artificiais dos ambientes telemáticos na construção de presença? De que maneira a interconectividade das redes e as mediações de distância pelas tecnologias da informação e comunicação contribuem para a emergência de uma experiência sensível? Como equalizar o nível de complexidade e sofisticação de ambientes telemáticos para se alcançar um equilíbrio entre as funções tecnológicas e as disfunções poéticas da arte? Se pensarmos os ambientes telemáticos como uma grande máquina complexa, talvez possamos aplicar as considerações de Gilbert Simondon sobre a margem de indeterminação (Simondon, 1989) necessária aos sistemas maquínicos para que eles se abram de forma mais produtiva e criativa aos processos de invenção aos quais os submetemos. Essas e muitas outras perguntas são sem dúvida parte de uma constelação de questões que tem emergido da prática ligada a criação de ambientes telemáticos e a interação entre seus agentes, sejam 
esses naturais ou artificiais. Certamente são perguntas sem respostas concretas pois vivemos o tempos dos fluxos contínuos, cuja a vertigem da aceleração tem substituído a nitidez das respostas fixas. Talvez a única certeza no horizonte dessa nova ecologia de homens e máquinas em fluxo seja a de que as redes são o tecido dessa nova cultura.

\section{Referências}

ARDUINO. Arduino. Disponível em: http://www.arduino.cc/. Data de acesso: 09/10/2006

GT de Mídias Digitais e Arte. Arthron: Uma Ferramenta para Performances Artístico-Midiáticas Distribuídas. Disponível em: http://www.lavid.ufpb.br/gtmda/Manual_Athron_ Final.pdf. Data de acesso: 10/01/2012

FLUSSER, V. Filosofia da caixa preta: ensaios para uma futura filosofia da fotografia. Rio de Janeiro: Relume Dumará. 2002

HO, M. W. The rainbow and the worm: the physics of organisms. Singapore; River Edge, NJ: World Scientific. 1993

NÓBREGA, C. Art and Technology: coherence, connectedness, and the integrative field. 2009. 297f. Ph.D-thesis. Planetary Collegium - School of Art and Media, University of Plymouth, Plymouth - UK.

. Thinking Hyperorganisms. Art, technology, coherence, connectedness, and the integrative field. Saarbrücken, Germany: LAP Lambert Academic Publishing. 2010

SIMONDON, G. Du mode d'existence des objets techniques. Paris: Aubier: Editions Montaigne. 1989

\section{NOTAS}

1. Cf. http://www.mapad2.ufba.br/laboratorium/

2. "A Arthron é uma ferramenta para facilitar a execução de performances artísticas que utilizam representações midiáticas e o compartilhamento de espaços reais e virtuais em tempo-real" (2005).

3. O conceito hiperorganismo aplicado aqui foi desenvolvido pelo autor em sua tese de doutoramento (Nóbrega, 2009) e em seu livro "Thinking Hyperorganisms. Art, technology, coherence, connectedness, and the integrative field" (Nóbrega, 2010).

4. "Breathing" é um sistema híbrido desenvolvido durante pesquisa de doutoramento na University of Plymouth, UK (Cf. www.gutonobrega.co.uk).

5. Trabalharam na programação do robô, assim como no design e implementação das vestes e produção geral os estudantes: Marlus Araújo (design de interatividade e programação), Leonardo Galvão (asssitência e pesquisa 
de interatividade), Danielle Uchoa (design de veste interativa), Brínea Costa (documentação e produção).

6. Para esse experimento foi utilizada a espécie Epipremnum Pinnatum, conhecida popularmente como "jibóia".

7. As variações em milivoltagens monitoradas pelo medidor GSR foram amplificadas por um circuito operacional e enviadas a um Arduino para processamento.

8. Light-Emitting-Diode capaz de combinar três fontes básicas de luzes coloridas (red, green, blue) para formar diversas cores do espectro visível a partir dessa combiação.

9. Tele-instrumento de sopro digital desenvolvido pelo músico, pesquisador e colaborador do projeto, Bruce Gremo.

10. Open Sound Control trata-se de um protocolo para comunicação entre computadores e outras fontes de dados numéricos através do uso otimizado de estruturas de redes (Cf. http://opensoundcontrol.org/introduction-osc).

11. Sistema de alta qualidade para streaming de audio bidirecionalmente, sem compressão, via internet (Cf. http://code.google.com/p/jacktrip/).

12. Por tecnicidade nos referimos a qualidades intríncicas aos objetos técnicos e seus elementos que acabam por informar estruturas complexas quando os mesmos são empregados como parte de sua construção . Por exemplo, o emprego de fibras óticas que são maleáveis e transparentes podem orientar a construção de sistemas que empregam esses materiais a se desenvolverem num sentido mais orgânico de suas formas.

13. Em vários momentos essas interrupções ocorreram, por diversos motivos. Uma das incompatibilidades dessa colaboração internacional foi o fato de China e Dinamarca trabalharem sob protocolo de rede IPV6, enquanto no Brasil trabalhávamos sob IPV4. Isso impossibilitou uma comunicação sonora via rede (Jack Trip) direta com a China. Uma ponte teve que ser feita com a Dinamarca (que operava tanto com IPV 4 como IPV6) para que obtivéssemos sucesso nas trocas de áudio, mas o sistema se mostrou instável.

Recebido em: 05/o1/12

Aceito em: 05/01/12

\section{GUTO NÓBREGA}

gutonobrega@gmail.com

Carlos Nóbrega (Guto Nóbrega) é Doutor (2009) em Interactive

Arts pelo programa de pós graduação Planetary Collegium (antigo

CAiiA-STAR), University of Plymouth UK. Guto Nóbrega é artista,

pesquisador, Mestre em Comunicação, Tecnologia e Estética pela ECO-

UFRJ (2003) e Bacharel em gravura pela EBA-UFRJ (1998) onde leciona

desde 1995 e atualmente fundou e coordena o NANO - Núcleo de Arte e

Novos Organismos. 